\title{
A novel RASA1 mutation causing capillary malformation-arteriovenous malformation (CM-AVM): the first genetic clinical report in East Asia
}

Ren Cai ${ }^{1,2 \dagger}$, Fatao $\mathrm{Liu}^{3,4 \dagger}$, Chen Hua ${ }^{1 \dagger}$, Zhang $\mathrm{Yu}^{1 \dagger}$, Michele Ramien ${ }^{5}$, Claudia Malic ${ }^{6}$, Wenxin $\mathrm{Yu}^{2}$, Xiaolin Zhang ${ }^{1}$, Yun Liu' ${ }^{7,8}$, Yunbo $\mathrm{Jin}^{1 *}$, Xun $\mathrm{Hu}^{9^{*}}$ and Xiaoxi Lin ${ }^{1,2^{*}}$

\begin{abstract}
Capillary malformation-arteriovenous malformation (CM-AVM) is a clinical entity newly identified in 2003 that is caused by mutation of the RASA-1 gene, which encodes the protein p120-RasGAP. To date, most of the clinical reports on CMAVM in the literature involve samples entirely consisting of Caucasians of European and North American descent, while reports from China or East Asia are few. Here, we describe a genetic clinical report of CM-AVM. Sequencing revealed a novel stop mutation in the RASA-1 gene causing loss of function (LOF) of the RasGAP domain. To our knowledge, this is the first genetic clinical report of a CM-AVM patient in East Asia. This report may extend our understanding and support further studies of CM-AVM in East Asia.
\end{abstract}

Keywords: Capillary malformation-arteriovenous malformation, RASA-1 mutation, RasGAP, China, East Asia

\section{Background}

Capillary malformations (CMs), or Port-wine stains (PWSs), are a form of vascular anomalies that are congenital, that progressively grow with the individuals, that do not regress spontaneously and that show normal rates of endothelial cell turnover [1-3]. They manifest as erythema that is pink-red in color, and they are related to skin and cutaneous tissue, usually located in the head and nuchal area, and are observed in approximately 3-5 out of every 1000 newborns [4]. In contrast, some atypical CMs occur in cutaneous zones as multiple small, white, pale halos with central red spots that are round to oval in shape [5, 6]. Importantly, these atypical CMs are hereditary in an autosomal

\footnotetext{
*Correspondence: docjinyunbo@163.com; zgyccb@163.com; linxiaoxi@126.com

${ }^{\dagger}$ Ren Cai, Fatao Liu, Chen Hua and Zhang Yu contributed equally to this work.

'Department of Plastic and Reconstructive Surgery, Shanghai Ninth People's Hospital, Shanghai Jiao Tong University School of Medicine, 639 Zhi Zao Ju Road, Shanghai 200011, People's Republic of China

${ }^{9}$ Department of Stomatology, Luwan Branch of Ruijin Hospital Affiliated to

Shanghai Jiao Tong University School of Medicine, Shanghai, People's

Republic of China

Full list of author information is available at the end of the article
}

dominant fashion, and observation allowed their mapping to the CMC1 locus on chromosome 5q13-22 [7, 8].

In 2003, Eerola et al. reported six families exhibiting this atypical CM, discovered its association with RASA-1 muatation, and defined this disorder as capillary malformation- arteriovenous malformation (CM-AVM) [5]. Given the localized nature of the vascular anomalies, a "second hit" hypothesis was proposed as the pathomechanism of the disorder $[5,6]$.

In addition to the multiple spots of erythema, fast-flow lesions such as arteriovenous malformation (AVM) or arteriovenous fistula (AVF) as well as hypertrophic syndromes such as Sturge-Weber syndrome (SWS) and Parker-Weber syndrome (PKWS) were also documented in families with a RASA-1 mutation [5, 9]. However, RASA-1 mutation was occasionally discovered in syndromes of overgrowth in length, for example, Klippel-Trenaunay syndrome (KTS) $[9,10]$.

CM-AVM indicates a high risk of fast-flow lesions. In a 2003 study, 6 out of 17 families manifested with atypical CM together with fast-low lesions such as AVM, AVF or PKWS [5]. Studies in 2008 and 2010 showed

(C) The Author(s). 2018 Open Access This article is distributed under the terms of the Creative Commons Attribution 4.0 International License (http://creativecommons.org/licenses/by/4.0/), which permits unrestricted use, distribution, and 
that approximately one-third of CM-AVM patients possess fast-flow vascular anomalies [9, 11-13].

RASA-1 encodes for Ras GTPase-activating protein 1, or p120-RasGTP-activating protein (p120-RasGAP), which is a negative regulator catalyzing the conversion of Ras to its GDP-bound form [14].

Mutation of GAP may affect the function of endothelial cells, leading them to form a highly vascularized network and resulting in extensive neuronal death [15]. Stimulation of annexin A6 regulates p120-RasGAP on the plasma membrane and hence controls the activity of Ras [16]. In a murine model experiment, RASA-1 ${ }^{+/-}$mice had a nor-

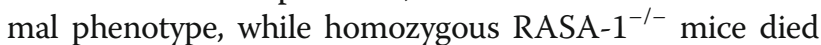
at E10.5 from the development of vascular dysfunction and increased neuronal apoptosis [15]. In the literature, most of the RASA-1 mutations associated with CM-AVM are deletions in the reading frame, causing a frame shift and subsequent a formation of a stop codon blocking further translation of the protein. These mutations result in a truncated protein with complete loss of function (LOF) of the RasGAP domain [5].

In this report, we describe two CM-AVM patients with a novel mutation in the RASA-1 gene $(\mathrm{c} .3070 \mathrm{~A}>\mathrm{T})$ leading to LOF of the p120-RasGAP domain. To our knowledge, this is the first report of CM-AVM related to a RASA-1 mutation in East Asia.

\section{Methods}

This study was conducted in accordance with the Declaration of Helsinki and was approved by the Ethics Committee of Shanghai Ninth People's Hospital Affiliated to Shanghai Jiaotong University School of Medicine (equivalent to an institutional review board). This study was performed according to the approved guidelines and regulations. Written informed consent for study participation and publication of identifying information and images was obtained from each subject and child's guardian prior to the study. A careful clinical record on the proband and his father was obtained by a medical geneticist and plastic surgeons.

\section{Clinical report}

The proband was a boy born at 39 weeks to a 27 -year-old G1P1 mother via a eutocia. His birth weight was $3600 \mathrm{~g}$, and his Apgar scores were 9 (0 mins) and 10 (5 mins). Both parents were of Chinese descent without consanguinity. In addition, no prenatal complications were noted by the parents.

The proband was born with small red lesions sporadically on his right parotid area. The lesions progressively spread to his right ear region. The skin temperature of the lesion was higher than that of the adjacent normal area. His pulse was detectable with palpation on the lesion. The ears of the proband appeared to be asymmetric (Fig. 1aA).
Physical examination revealed multiple spots of erythema in different areas of the body (Additional file 1). He had a large lesion extending from his right cheek to his ear and neck (Fig. 1aB-C). Additionally, there were some oval to round spots of erythema in his left arm and left knee (Fig. 1aD-E). From the family history, the proband's father had an identical pattern of multiple spots of erythema on the neck, abdomen, back, waist, elbow and right D5 dermatome (Fig. 1aF-L). Multifocal erythema was not observed in other family members.

\section{Magnetic resonance imaging (contrast-enhanced)}

MRI was performed using a $3.0 \mathrm{~T}$ MRI unit (MAGNETOM Verio, Siemens, Germany) with a head and neck coil. The MRI protocol included unenhanced axial and coronal T2-weighted sequences, axial T1-weighted sequences, and contrast-enhanced fat-suppressed axial, sagittal and coronal T1-weighted sequences. The parameters for these sequences were as follows: T1-weighted spin echo (SE) sequence $=620-\mathrm{ms}$ repetition time $(\mathrm{TR}), 8.2-\mathrm{ms}$ echo time (TE), 4-mm section thickness, 260-mm*260-mm field of view, 256*256 matrix; T2-weighted turbo-spin echo sequence $=4500-\mathrm{ms} \mathrm{TR}, 80-\mathrm{ms}$ TE, 4-mm section thickness, 260-mm*260-mm field of view, $256 * 256$ matrix.

\section{DNA extraction}

Genomic DNA was extracted from peripheral blood by using a QIAamp DNA Blood Mini Kit (QIAGEN) according to the manufacturer's instructions. A skin biopsy was performed on the CM-AVM lesion of the proband via a standard procedure. DNA of biopsy tissues was extracted by using the QIAamp DNA Mini Kit (QIAGEN) with overnight incubation in proteinase $\mathrm{K}$ at $56^{\circ} \mathrm{C}$ for approximately $14 \mathrm{~h}$. A Qubit 2.0 fluorimeter (Life Technologies, Carlsbad, CA, USA) and a Thermo NanoDrop 2000 spectrophotometer (Thermo, Wilmington, DE, USA) were used to determine the DNA concentration and quality.

\section{Next-generation sequencing (NGS) and Bioinformatic analysis}

To target the exons as well as the corresponding boundary regions, a series of RNA baits for RASA-1 were designed and synthesized. We used the SureSelectXT kit reagents (Agilent Technologies, Santa Clara, CA) for the Illumina adapters. The biotinylated RNA baits were applied in hybridization reactions at $65^{\circ} \mathrm{C}$ for $24 \mathrm{~h}$ to generate the adapter-ligated DNA libraries. Then, streptavidin-coated magnetic beads were used to capture the hybridized DNA targets. The target DNA was washed, eluted, and finally barcoded. An Agilent 2100 Bioanalyzer (Thermo) was used to determine the length and integrity of the library.

Quantitative PCR (KAPA Biosystems, Wilmington, MA) was applied to verify the concentration of the indexed libraries, after which the libraries were sequenced on a 

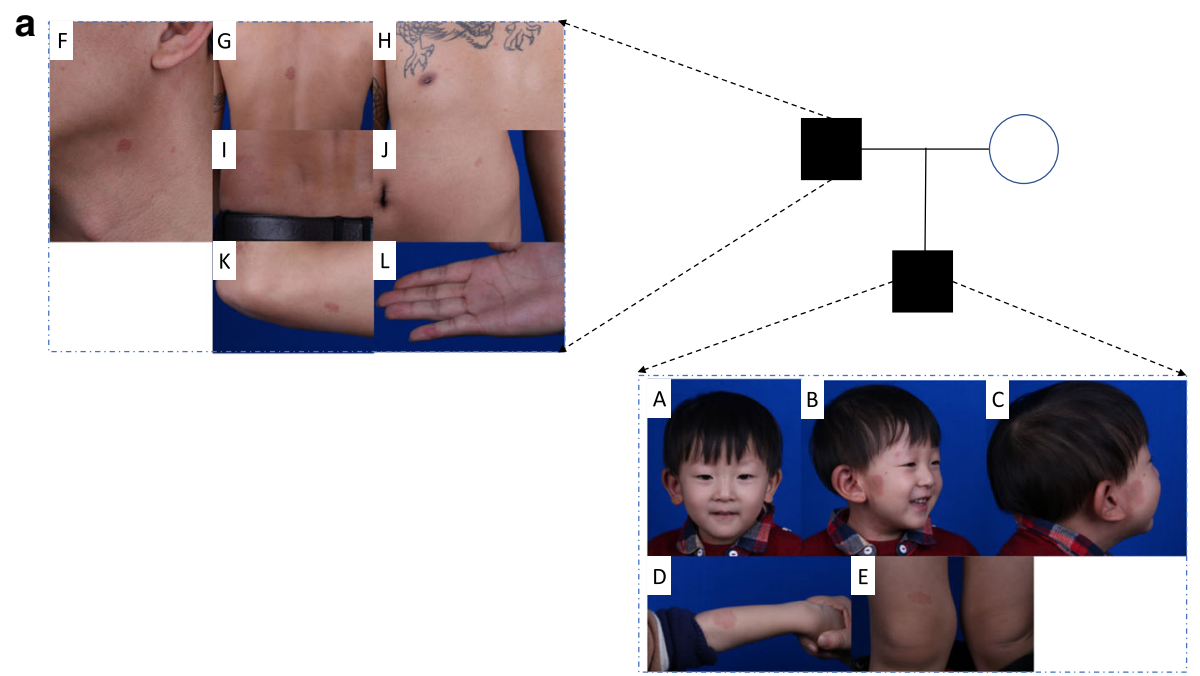

b

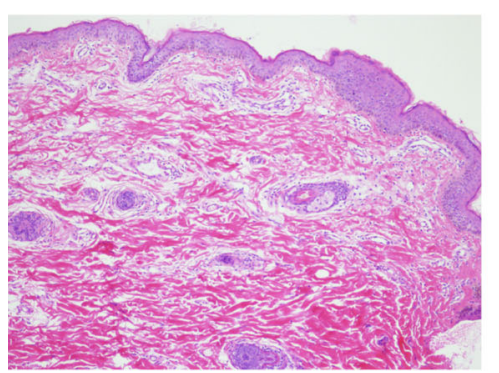

C

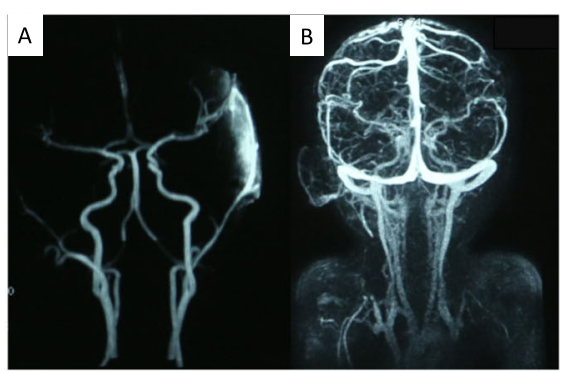

Fig. 1 Clinical Manifestations, pathology finds and MRA images of the Proband's Family. a: A: Asymmetric appearance of the ears of the proband. B: Erythema on the head and neck of the proband. C: Erythema on the right parotid area and ear of the probandD: Erythema on the left forearm of the proband. E: Erythema on the right knee of the proband. F: Erythema on the neck of the proband's father. G: Erythema on the back of the proband's father. H: Erythema on the chest of the proband's father. I: Erythema on the waist of the proband's father. J: Erythema on the abdomen of the proband's father. K: Erythema on the elbow of the proband's father. L: Erythema on the right D5 dermatome of the proband's father. $\mathbf{b}$ : Hypertrophy in the epidermis and small, tufted capillary malformations in the superficial dermis (100X, HE). c: Abnormal arterial networks from the ECA in his right auricular region and neck cutaneous tissue

MiSeq instrument (Illumina, San Diego, CA). The resulting sequencing reads were trimmed with known adaptors, quality-checked and then mapped to a human reference genome (hg19, downloaded from http://genome.ucsc.edu) by using Burrows-Wheeler Aligner (BWA 0.5.9, http:// bio-bwa.sourceforge.net) with the default parameters. PCR duplicates were marked and removed by using PICARD software. Additionally, Genome Analysis Toolkit (GATK, https://www.broadinstitute.org/gatk/) was applied to conduct indel realignment, base recalibration and variant calling. The resulting .vcf files were then annotated by VEP software (http://www.ensembl.org/info/docs/tools/vep/) and the GEMINI framework (http://gemini.readthedocs.io/ en/latest/index.html). By using Integrative Genomic Viewer (IGV) software, variants were visualized and checked.

\section{Sanger sequencing}

RASA1 exon 25 was PCR-amplified to track the c.3070A > T (p.Lys1024*) variant in the blood and tissue samples from the family. Primer sequences are available upon request. Amplicon fragments were sequenced bidirectionally (forward and reverse) with the M13 primer by the BigDye Terminator v3.1 cycle sequencing kit and an ABI 3730 DNA Analyzer (Life Technologies, Carlsbad, CA). Target sequences were compared to the RASA1 reference sequence (NM_002890) using Mutation Surveyor (SoftGenetics, State College, PA) (Additional file 2).

\section{Results}

Molecular analysis of the DNA extracted from the peripheral blood of the proband and the proband's parents confirmed that the proband's father and the proband harbored a familial germline mutation (c.3070A > T, p.Lys1024*) in the RASA-1 gene (Figs. 2 and 3). NGS analysis showed a depth of coverage for this mutation of $16,260 \times$ and $16,451 \times$ in blood samples (Table 1). This mutation was also detected in the proband's skin lesion at a depth of coverage of 21,026× (Table 1). The score of this RASA-1 germline mutation (c.3070A $>$ T, p.Lys1024*) from the 


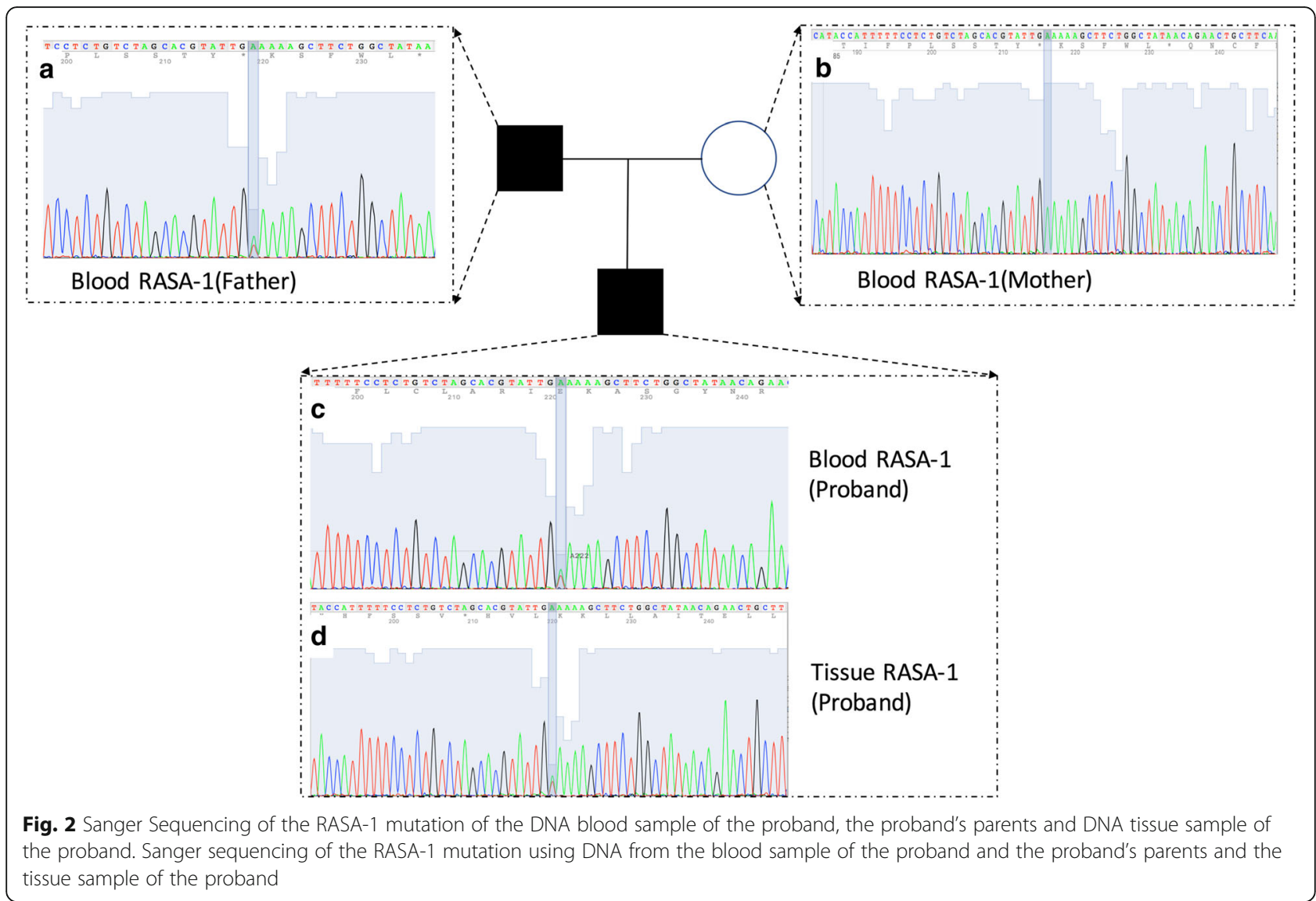

1000 Genomes database of East Asia was - 1, indicating that the mutation had never been reported before.

Radiologic Assessment: Contrast-enhanced MRI demonstrated that there were abnormal arterial networks from the external carotid artery (ECA) in his right auricular region and neck cutaneous tissue (Fig. 1c). Because of the harm of radiation, we did not perform digital subtraction angiography (DSA)on the child (Fig. 1cA-B).

Pathologic Findings: Under the microscope, hyperplasia was observed in the epidermis showed hyperplasia, small tufted vessels were observed in the superficial dermis, and ectatic vessels were observed in the deep dermis (Fig. 1b, HE, 100x).

\section{Discussion}

The proband and his father were diagnosed with CM-AVM based on their clinical manifestations according to the diagnostic criteria [17]. Suspicions of CM-AVM were raised due to the multifocal cutaneous erythema observed even without the fast-flow lesions [13]. Findings of congenital and acquired skin lesions that were round to oval in shape and $1-3 \mathrm{~cm}$ in diameter and that had small anemic halos confirmed the disorder at the clinical level $[5,6,18,19]$. In addition, the LOF mutation of RASA-1 enabled a definite diagnosis at the genetic level. MRI demonstrated abnormal networks from the ECA, which indicated that the patients had the potential for AVM invasion.

According to the literature, CM-AVM patients have a high risk of high-flow lesions such as AVM and AVF [9, 11-13], which can affect soft tissues such as dermis, muscle and even central nervous system tissues, leading to anomalies, malformations, dysfunction or disabilities [20]. In this case, according to the results of MRI, the patient had the potential for AVM invasion. Precautions such as regular clinical follow-up and bleomycin injection should be taken to prevent AVM formation [21].

RASA1 facilitates the inactivation of Ras p21 by enhancing the GTPase activity of Ras proteins [22]. LOF mutation of RASA-1 may have numerous consequences on downstream signaling, such as aberrant proliferation, angiogenesis [23] and increased ERK activation in vitro [24]. The mutation of RASA-1 in this case was the base substitution c.3070A $>$ T (p.Lys1024\%, Fig. 3) present in the RasGAP domain halting further translation, leading to the LOF of the RASA1 gene.

The association between the phenotype and the genotype of the RASA-1 mutation in CM-AVM is still unclear. The genotype-phenotype relationship and its 


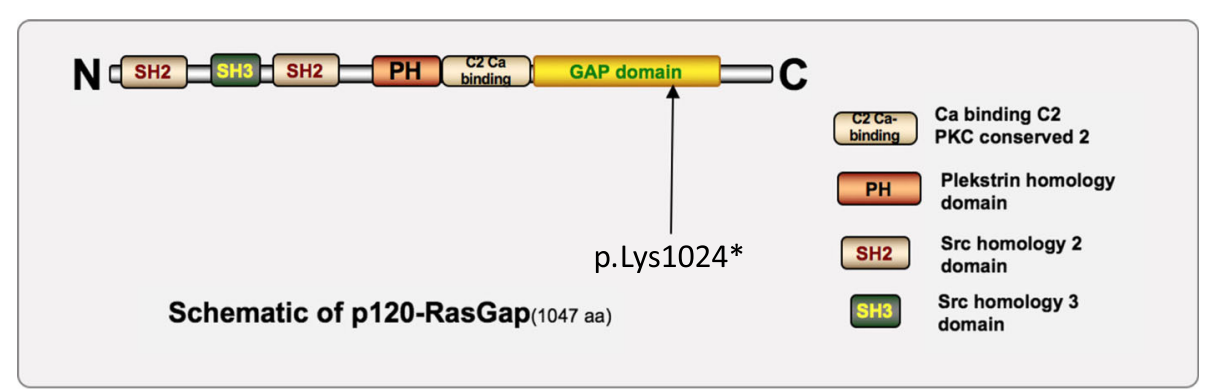

Fig. 3 Schematic of p120-RasGAP. Schematic of p120-RasGAP, the 1047-amino-acid protein encoded by the RASA-1 gene. With two Src homology $2(\mathrm{SH} 2)$ domains and one Src homology $3(\mathrm{SH} 3)$ domain in the N-terminal region, a Pleckstrin homology $(\mathrm{PH})$ domain and a protein kinase conserved region 2 in the central region (C2), and a Ras GTPase-activating domain in the C-terminal region (RasGAP), p120-GAP negatively regulates the Ras/MAPK pathway. Our patient and his father harbored a mutation (c.3070A > T, p.Lys1024*), labeled with the solid arrow, halting the further translation of p120-RasGAP, which consequently led to CM-AVM

mechanisms have not been well elucidated, even though there are some hypothesized mechanisms, such as changes in the expression pattern of different growth factors due to the RASA-1 mutation [5]. Another proposal is the somatic "second-hit" hypothesis, in which fast-flow lesions may form because of haploinsufficiency of the RASA-1 mutation when a specific population of cells experience complete LOF caused by a "second-hit" somatic mutation in addition to the "first-hit" germline mutation $[6,11]$.

However, in this case, did we not detect any additional mutations on the RASA-1 gene to support the "second-hit" hypothesis, nor did we detect a somatic mutation of GNAQ, which is observed in $88 \%$ of SWS cases and $92 \%$ of apparently nonsyndromic PWS cases [25]. Thus, we can differentiate CM-AVM from nonsyndromic PWSs at the genetic level. The specific cell type harboring the mutation is not known and still requires further study.

The phenotype of this CM-AVM family was discordant. The proband exhibited multifocal erythema and potential AVM invasion from the ECA, while his father exhibited multifocal erythema without any high-flow lesions. We do not know whether there was intrafamilial variability in RASA-1 mutation-related conditions in this family, and further study is needed to clarify this issue.

This study emphasizes the importance of NGS, together with a careful medical history and good cooperation with the radiology department, for facilitating the discovery of new clinical findings. As plastic surgeons or dermatologists, we can easily overlook or neglect multifocal erythema and choose laser therapy as the treatment, which may lead to a great risk when the erythema is part of a CM-AVM lesion with underlying AVM. The proband will receive genetic testing; know the potential risk of fast-flow lesion formation; have regular clinical follow-ups to prevent lesion formation or obtain appropriate initial treatment; and take precautions against the life-threating complications of AVM or AVF, such as hemorrhage, congestive heart failure, and seizures [5, 11, 12, 17].

Table 1 NGS Genetic Information of mutation of proband and proband's father

\begin{tabular}{llll}
\hline DNA Sample & Blood of the Proband's Father & Blood of the Proband & Tissue of the Proband \\
\hline gene & RASA1 & RASA1 & RASA1 \\
chr:posi & $5: 86686626$ & $5: 86686626$ & $5: 86686626$ \\
ref $>$ alt & A $>$ T & A $>$ T & A T T \\
type & SNV & SNV & SNV \\
geno & $0 / 1$ & $0 / 1$ & $0 / 1$ \\
AD & $8787+7664$ & $8491+7769$ & $10,029+10,997$ \\
hgvs_c & C.3070A $>$ T & C.3070A $>T$ & C.3070A > T \\
hgvs_P & p.Lys1024* & p.Lys1024* & p.Lys1024* \\
impact & Stop gained & stop gained & stop gained \\
impact_severity & HIGH & HIGH & HIGH \\
aaf_1kg_eas & None & None & None \\
in_exac & None & None & None \\
\hline
\end{tabular}




\section{Conclusion}

The mutation of RASA-1 in this patient is the base substitution c.3070A > T (p.Lys1024*) present in the RasGAP domain halting further translation. To our knowledge, this is the first description of this mutation in East Asia. It would be valuable to measure the Ras/ MAPK activity level in vitro, such as in HUVECs from the samples collected from the patient and his father. Such information would help us understand the angiogenesis of CM-AVM, especially for patients in East Asia.

\section{Additional files}

Additional file 1: Cutaneous findings of the proband's family. (DOC 37 $\mathrm{kb})$

Additional file 2: Information about the primers used to detect the c.3070A > T (p.Lys1024*) variant. (DOC 35 kb)

\section{Abbreviations}

AVF: Arteriovenous Fistula; AVM: Arteriovenous Malformation; CM: Capillary Malformation; CM-AVM: Capillary Malformation-Arteriovenous Malformation; ECA: External Carotid Artery; KTS: Klippel-Trenaunay Syndrome; LOF: Loss-ofFunction; MRI: Magnetic Resonance Imaging; PKWS: Parker-Weber Syndrome; PWS: Port-Wine Stain; SWS: Sturge-Weber Syndrome

\section{Acknowledgements}

We sincerely thank the family for their participation and their noble cooperation with our clinical and laboratory work. And thank Jessica Zeng for her company and assistance in spirit.

\section{Funding}

This work was supported by the National Natural Science Foundation of China (No. 81602777).

\section{Availability of data and materials}

The datasets generated during and analyzed during the current study are available from the corresponding author on reasonable request.

\section{Authors' contributions}

$R C, F L, C H, Z Y, W Y, X Z$ and $X L$ participated in the treatment of the patient at our Vascular Anomalies Center and in designing the clinical report of this patient. The majority of the original draft of the paper was written by RC, FL, and $\mathrm{CH}, \mathrm{RC}$ and $\mathrm{CH}$ carried out next-generation sequencing of the patient's blood and tissue samples, and FL carried out the bioinformatics analysis. XZ recorded the clinical follow-up. $\mathrm{XH}$ participated in the biopsy on the patient. MR and CM participated in writing and revising the paper. All authors reviewed the manuscript. All authors read and approved the final manuscript.

\section{Ethics approval and consent to participate}

Approved by the Ethics Committee of Shanghai Ninth People's Hospital Affiliated to Shanghai Jiaotong University School of Medicine (equivalent to an institutional review board).

\section{Consent for publication}

Acquired.

\section{Competing interests}

The authors declare that they have no competing interests.

\section{Publisher's Note}

Springer Nature remains neutral with regard to jurisdictional claims in published maps and institutional affiliations.

\section{Author details}

'Department of Plastic and Reconstructive Surgery, Shanghai Ninth People's Hospital, Shanghai Jiao Tong University School of Medicine, 639 Zhi Zao Ju Road, Shanghai 200011, People's Republic of China. ${ }^{2}$ Department of Laser and Cosmetic Surgery, Shanghai 9th People's Hospital, Shanghai, People's Republic of China. ${ }^{3}$ Department of General Surgery, Xinhua Hospital Affiliated to Shanghai Jiao Tong University School of Medicine, Shanghai, People's Republic of China. ${ }^{4}$ Institute of Biliary Tract Disease, Shanghai Jiao Tong University School of Medicine, Shanghai, People's Republic of China. ${ }^{5}$ Department of Dermatology, Children's Hospital of Eastern Ontario, Ottawa, Canada. ${ }^{6}$ Department of Surgery, Children's Hospital of Eastern Ontario, Ottawa, Canada. ${ }^{7}$ Institutes of Biomedical Sciences, Fudan University, Shanghai, People's Republic of China. ${ }^{8}$ Bio-X Institutes, Key Laboratory for the Genetics of Developmental and Neuropsychiatric Disorders (Ministry of Education), Shanghai Jiao Tong University, Shanghai, People's Republic of China. ${ }^{9}$ Department of Stomatology, Luwan Branch of Ruijin Hospital Affiliated to Shanghai Jiao Tong University School of Medicine, Shanghai, People's Republic of China.

Received: 2 April 2018 Accepted: 11 June 2018

Published online: 16 July 2018

\section{References}

1. Jacobs AH, Walton RG. The incidence of birthmarks in the neonate. Pediatrics. 1976;58:218-22.

2. Mulliken JB, Young AE. Vascular birthmarks: hemangiomas and malformations. Philadelphia: WB Saunders Company; 1988.

3. Pratt AG. Birthmarks in infants. AMA Arch Derm Syphilol. 1953;67:302.

4. Wassef M, Blei F, Adams D, Alomari A, Baselga E, Berenstein A, et al. Vascular anomalies classification: recommendations from the International Society for the Study of vascular anomalies. Pediatrics. 2015;136:e203-14. https://doi. org/10.1542/peds.2014-3673.

5. Eerola I, Boon LM, Mulliken JB, Burrows PE, Dompmartin A, Watanabe S, et al. Capillary malformation - arteriovenous malformation, a new clinical and genetic disorder caused by RASA1 mutations. Am J Hum Genet. 2003;73: 1240-9

6. Revencu N, Boon LM, Mendola A, Cordisco MR, Dubois J, Clapuyt P, et al. RASA1 mutations and associated phenotypes in 68 families with capillary malformation-arteriovenous malformation. Hum Mutat. 2013:34:1632-41. https://doi.org/10.1002/humu.22431.

7. Breugem CC, Alders M, Salieb-Beugelaar GB, Mannens MM, Van der Horst CM, Hennekam RC. A locus for hereditary capillary malformations mapped on chromosome 5q. Hum Genet. 2002;110:343-7. https://doi.org/10.1007/ s00439-002-0700-z.

8. Eerola I, Boon LM, Watanabe S, Grynberg H, Mulliken JB, Vikkula M. Locus for susceptibility for familial capillary malformation ('port-wine stain') maps to 5q. Eur J Hum Genet. 2002;10:375-80. https://doi.org/10.1038/sj.ejhg. 5200817.

9. Hershkovitz D, Bergman R, Sprecher E. A novel mutation in RASA1 causes capillary malformation and limb enlargement. Arch Dermatol Res. 2008;300: 385-8. https://doi.org/10.1007/s00403-008-0842-5.

10. Revencu N, Boon LM, Dompmartin A, Rieu P, Busch WL, Dubois J, et al. Germline mutations inRASA1 are not found in patients with KlippelTrenaunay syndrome or capillary malformation with limb overgrowth. Mol Syndromol. 2013;4:173-8. https://doi.org/10.1159/000349919.

11. Revencu N, Boon LM, Mulliken JB, Enjolras O, Cordisco MR, Burrows PE, et al, Parkes Weber syndrome, vein of Galen aneurysmal malformation, and other fast-flow vascular anomalies are caused by RASA1 mutations. Hum Mutat. 2008;29:959-65. https://doi.org/10.1002/humu.20746.

12. Thiex R, Mulliken JB, Revencu N, Boon LM, Burrows PE, Cordisco M, et al. A novel association between RASA1 mutations and spinal arteriovenous anomalies. AJNR Am J Neuroradiol. 2010;31:775-9. https://doi.org/10.3174/ajnr.A1907.

13. Hershkovitz D, Bercovich D, Sprecher E, Lapidot M. RASA1 mutations may cause hereditary capillary malformations without arteriovenous malformations. Br J Dermatol. 2008;158:1035-40. https://doi.org/10.1111/j. 1365-2133.2008.08493.x.

14. Pamonsinlapatham $P$, Hadj-Slimane $R$, Lepelletier $Y$, Allain $B$, Toccafondi M Garbay C, et al. p120-Ras GTPase activating protein (RasGAP): a multiinteracting protein in downstream signaling. Biochimie. 2009:91:320-8. https://doi.org/10.1016/j.biochi.2008.10.010. 
15. Henkemeyer M, Rossi DJ, Holmyard DP, Puri MC, Mbamalu G, Harpal K, et al. Vascular system defects and neuronal apoptosis in mice lacking ras GTPaseactivating protein. Nature. 1995;377:695-701. https:/doi.org/10.1038/377695a0.

16. Grewal T, Evans R, Rentero C, Tebar F, Cubells L, de Diego I, et al. Annexin A6 stimulates the membrane recruitment of p120GAP to modulate Ras and Raf-1 activity. Oncogene. 2005;24:5809-20. https://doi.org/10.1038/sj.onc.1208743.

17. Orme CM, Boyden LM, Choate KA, Antaya RJ, King BA. Capillary malformationarteriovenous malformation syndrome: review of the literature, proposed diagnostic criteria, and recommendations for management. Pediatr Dermatol. 2013;30:409-15. https:/doi.org/10.1111/pde.12112.

18. Gandon C, Bonniaud B, Collet E, Dalac S, Jeudy G, Vabres P. A typical vascular and pigmentary dermoscopic pattern of capillary malformations in capillary malformation-arteriovenous malformation syndrome: report of four cases. Pediatr Dermatol. 2016;33:e337-41. https://doi.org/10.1111/pde.12937.

19. Happle R. The rhodoid nevus: a proposed term for a so far unnamed capillary malformation. Dermatology. 2010;221:317-9. https://doi.org/10. 1159/000321331.

20. Bayrak-Toydemir P, Stevenson D. RASA1-related disorders. In: Pagon RA, Adam MP, Ardinger HH, Wallace SE, Amemiya A, Bean $L J H$, et al., editors. GeneReviews(R). Seattle: University of Washington Press; 1993.

21. Jin Y, Zou Y, Hua C, Chen H, Yang X, Ma G, et al. Treatment of early-stage extracranial arteriovenous malformations with intralesional interstitial bleomycin injection: a pilot study. Radiology. 2018;287:194-204. https://doi. org/10.1148/radiol.2017162076

22. Trahey M, McCormick F. A cytoplasmic protein stimulates normal N-ras p21 GTPase, but does not affect oncogenic mutants. Science (New York, NY). 1987;238:542-5.

23. Anand S, Majeti BK, Acevedo LM, Murphy EA, Mukthavaram R, Scheppke L et al. MicroRNA-132-mediated loss of p120RasGAP activates the endothelium to facilitate pathological angiogenesis. Nat Med. 2010;16:90914. https://doi.org/10.1038/nm.2186

24. Norden PR, Kim DJ, Barry DM, Cleaver OB, Davis GE. Cdc42 and k-ras control endothelial tubulogenesis through apical membrane and cytoskeletal polarization: novel stimulatory roles for GTPase effectors, the small GTPases, Rac2 and Rap1b, and inhibitory influence of Arhgap31 and Rasa1. PLoS One. 2016;11:e0147758. https://doi.org/10.1371/journal.pone.0147758.

25. Shirley MD, Tang H, Gallione CJ, Baugher JD, Frelin LP, Cohen B, et al. Sturge-Weber syndrome and port-wine stains caused by somatic mutation in GNAQ. N Engl J Med. 2013;368:1971-9. https://doi.org/10.1056/ NEJMoa1213507.

Ready to submit your research? Choose BMC and benefit from:

- fast, convenient online submission

- thorough peer review by experienced researchers in your field

- rapid publication on acceptance

- support for research data, including large and complex data types

- gold Open Access which fosters wider collaboration and increased citations - maximum visibility for your research: over $100 \mathrm{M}$ website views per year

At BMC, research is always in progress.

Learn more biomedcentral.com/submissions 